\title{
TITLE:
}

\section{Influence of gravity on collisions of monodispersed droplets in homogeneous isotropic turbulence}

\author{
AUTHOR(S):
}

Onishi, Ryo; Takahashi, Keiko; Komori, Satoru

\section{CITATION:}

Onishi, Ryo ... [et al]. Influence of gravity on collisions of monodispersed droplets in homogeneous isotropic turbulence. PHYSICS OF FLUIDS 2009, 21(12): 125108.

\section{ISSUE DATE:}

2009-12

URL:

http://hdl.handle.net/2433/109891

\section{RIGHT:}

Copyright 2009 American Institute of Physics. This article may be downloaded for personal use only. Any other use requires prior permission of the author and the American Institute of Physics. The following article appeared in PHYSICS OF FLUIDS 21, 125108 (2009) and may be found at http://link.aip.org/link/PHFLE6/V21/i12/p125108/s1 


\title{
Influence of gravity on collisions of monodispersed droplets in homogeneous isotropic turbulence
}

\author{
Ryo Onishi, ${ }^{1, a)}$ Keiko Takahashi, ${ }^{1}$ and Satoru Komori ${ }^{2}$ \\ ${ }^{1}$ Earth Simulator Center, Japan Agency for Marine-Earth Science and Technology, 3173-25 Showa-machi, \\ Kanazawa-ku Yokohama Kanagawa 236-0001, Japan \\ ${ }^{2}$ Department of Mechanical Engineering, Kyoto University, Kyoto 606-8501, Japan
}

(Received 6 August 2009; accepted 18 November 2009; published online 30 December 2009)

\begin{abstract}
This paper studies the gravity influence on collisions of monodispersed droplets in homogeneous isotropic turbulence by means of direct numerical simulations (DNSs). The DNS results show that, in certain Stokes and Reynolds regimes, collision frequencies are significantly reduced in the presence of gravity. Those decreases are mainly attributable to the decrease in the droplet relative velocity, since the change in radial distribution function-often referred to preferential concentration-is small. Further analysis of the results reveals that droplet sedimentation due to gravity shortens the droplet-fluid interaction time, consequently weakening the relative motions between droplets. These observations lead to an analytical model that can be used to estimate the velocity fluctuations of sedimenting particles under gravity. Utilizing this model, we constructed a further analytical model for estimating the gravitational influence on collisions. Given flow and particle parameters, the model calculates the ratio of collision frequencies with and without the effect of gravity. Past studies simply noted that the gravitational influence is negligible when the droplet sedimenting velocity is much smaller than the flow velocity fluctuations. Our analytical model further suggests that the gravitational influence on collisions of monodispersed cloud droplets with non-negligible sedimentation rates stays negligibly small even in high Reynolds number flows, such as those typically found in convective clouds. (c) 2009 American Institute of Physics.
\end{abstract}

[doi:10.1063/1.3276906]

\section{INTRODUCTION}

Following their formation through nucleation, cloud particles proceed to grow by the process of vapor diffusion, i.e., by condensation. The initial stage of droplet growth by condensation produces a fairly uniform distribution, with droplets generally between 1 and $10 \mu \mathrm{m}$ in radius. Further broadening of the spectrum toward precipitation-sized droplets (radii $\geq 100 \geqslant \mu \mathrm{m}$ ) is possible only when in-cloud conditions allow droplets to collide and coalesce. However, the conventional understanding of the collision process has not been sufficient to explain the fast growth of cloud droplets across the size gap from 10 to $50 \mu \mathrm{m}$ in radius, which results in the fast initiation of warm rain (e.g., Ref. 1). Various ideas have been proposed to explain the discrepancies between theory and observations. For example, entrainment and mixing of dry air by large scale turbulence (e.g., Refs. 2 and 3), the existence of ultragiant cloud condensation nuclei (e.g., Refs. 4 and 5) and nonuniformity of the supersaturation field (e.g., Ref. 6) may lead to enhanced droplet growth in the condensational phase of rain formation. The general hypothesis, however, is that the interaction between in-cloud turbulence and droplet dynamics can strongly affect the collision frequency. As a result, turbulence may significantly enhance droplet growth in the collision-coalescence stage of warm rain formation.

Reviews of the interactions between turbulence and

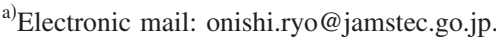

droplets in clouds ${ }^{7-11}$ mention at least three mechanisms that significantly affect the collision-coalescence frequency of cloud droplets. First, relative velocities between colliding droplets are increased because of the varying drag forces that droplets experience, leading to large variations in droplet velocities. Moreover, the relative velocities of sedimenting droplets can be changed by turbulence through an increase in average settling velocities, a phenomenon known as preferential sweeping. ${ }^{12-14}$ Second, droplets are centrifuged out of vortical structures through their finite inertia, and hence accumulate in regions with low vortical activity. This local clustering of droplets is known as preferential concentration. ${ }^{15-17}$ Third, the collision efficiency ${ }^{18}$ needs to be modified in the presence of turbulence due to the more complex behavior of local hydrodynamic droplet-droplet interactions. ${ }^{19,20}$

Over the last decade, increased computational power made it possible to study these microphysical processes in more detail by means of direct numerical simulations (DNSs). The major advantage of DNS is that it explicitly solves the smallest turbulent scales, where most of the interaction with droplet dynamics takes place. ${ }^{10}$ Franklin et $a l .^{21,22}$ employed DNS in the context of cloud microphysics and found turbulence-induced increases of the collision frequency to be dependent on the dissipation rate. They also showed that these increases originate from preferential concentration and increased relative velocities. Corresponding results were obtained by Wang et al., ${ }^{18,19}$ who also included hydrodynamic effects in their DNS to study its effect on 
droplet clustering, relative velocities, and settling velocities. In a theoretical study, Ghosh et al. ${ }^{23}$ investigated the effect of increased settling velocities in the context of fast rain initiation.

Apart from the above-mentioned collision efficiencies due to droplet-droplet hydrodynamic interactions, the collision-coalescence process is largely determined by the geometric collision kernel, which measures the collision frequency from the geometric overlap between droplets. In the past, various attempts have been undertaken to model the geometric collision kernel in a turbulent flow. Saffman and Turner $^{24}$ developed an expression for small-inertia particles in turbulence that was corrected and extended by Wang et $a l^{25}$ using a spherical formulation. Dodin and Elperin ${ }^{26}$ corrected the Saffman and Turner expression to be valid for the limiting cases of inertialess particles without gravity and sedimenting particles without turbulence. However, neither study included the influence of droplet clustering or the coupling between gravity and turbulence, which can be of major importance as pointed out by, e.g., Grabowski and Vaillancourt. ${ }^{27}$ Wang et $a l_{.}{ }^{28}$ and Zhou et al. ${ }^{29}$ developed models for turbulent collisions in the case of monodispersed and bidispersed droplets, respectively. However, both models are based on DNS results that used frozen flow fields without gravity. The use of these models in a recent cloud physics study stimulated a discussion within the cloud microphysics community. ${ }^{30,31}$ For example, Franklin et al. ${ }^{21}$ showed that the use of a frozen flow field leads to overestimation of the collision kernel by up to $30 \%$ compared to when the flow field is allowed to evolve. In conclusion, there is still a need for a physically based model for the geometric collision kernel that includes the effects of both turbulence and gravity.

There are two kinds of gravity contributions to collisions. In the presence of gravity, collisions between different-sized droplets are caused by the difference in their settling velocities (hereafter referred to as the differential settling contribution). Gravitational sedimentation influences the droplet-fluid interaction, and consequently changes the collision frequency (hereafter referred to as the gravitational aerodynamic contribution). Collisions between monodispersed droplets are induced by a combination of turbulent collisions and the gravitational aerodynamic contribution, while those between bidispersed droplets additionally include the differential settling contribution. Although several collision kernel models are now available, all of them neglect the gravitational aerodynamic contribution (Refs. 28 and 32 for monodispersed droplets; Refs. 18, 29, and 31 for bidispersed droplets).

One consensus concerning the gravitational aerodynamic contribution is that it can be neglected when the droplet sedimentation velocity is negligible compared to the fluid velocity fluctuations. ${ }^{9,10,33}$ However, it is not well understood how the aerodynamic contribution affects the turbulent collisions of droplets when sedimentation is non-negligible. Wang and Maxey $^{12}$ studied the aerodynamic contribution to turbulent collisions of monodispersed droplets and found it to slightly decrease the collision frequency. Recently, Woittiez et al. ${ }^{34}$ investigated the aerodynamic contribution to turbulent collisions of bidispersed droplets as well as monodispersed drop- lets. They observed that it increases the collision frequency for $r>40 \mu \mathrm{m}$, but decreases it slightly for $r<40 \mu \mathrm{m}$. They also observed a large influence on the amount of preferential concentrations for both monodispersed case and bidispersed cases. No studies, however, discussed the Reynolds dependency of the aerodynamic contribution.

This study therefore aims to investigate the Reynolds dependency of the gravitational aerodynamic contribution to turbulent collisions by means of DNS of particle-immersed homogeneous isotropic turbulence. As an idealized condition, we use monodispersed droplets targeting the early stage of collisional growth, where a fairly uniform distribution is produced by condensational growth. A limitation of employing DNS is that it cannot resolve the wide range of turbulent scales typically found in typical environmental flows. To overcome this limitation, we also construct a model for estimating the aerodynamic contribution as a function of flow Reynolds number and droplet size. This model enables us to make a conjecture about the aerodynamic contribution in environmental flows, such as in-cloud turbulent flows.

In the next section, we describe our numerical method. Simulation results are presented in Sec. III A, and the model for estimating the gravitational aerodynamic contribution proposed and validated in Secs. III B and III C. Discussion of the gravitational influence in high Reynolds number flows is presented in Sec. III D. Conclusions are presented in Sec. IV.

\section{DIRECT NUMERICAL SIMULATION OF DROPLET-IMMERSED HOMOGENEOUS ISOTROPIC TURBULENCE}

\section{A. Flow field}

A pseudospectral method based on the Fourier-Galerkin method was used to solve three dimensional vorticity equations:

$$
\frac{\partial \omega_{i}}{\partial t}+U_{j} \frac{\partial \omega_{i}}{\partial x_{j}}=\omega_{j} \frac{\partial U_{i}}{\partial x_{j}}+\nu \frac{\partial^{2} \omega_{i}}{\partial x_{j} \partial x_{j}}+F_{i} .
$$

Here, $U_{i}$ is the air velocity in the $x_{i}$-direction, $\omega_{i}$ is the vorticity around the $x_{i}$-axis, and $F_{i}$ represents an external force. The gravitational force is not included in the flow equation since buoyancy effects are not targeted in this study. The kinematic viscosity, $\nu$, was set to $1.50 \times 10^{-5} \mathrm{~m}^{2} / \mathrm{s}$ at 1 atm and $298 \mathrm{~K}$. A forcing scheme for the low-wavenumber range was used to generate steady flows. ${ }^{15}$ In this scheme, a nonuniform, time-independent, large-scale force field was applied to the flow at each time step. The fourth-order RungeKutta method was used to simulate time evolutions, while the two-thirds method was used to eliminate aliasing errors. The vorticity equations were discretized on a cubic domain of length $2 \pi L_{0}$, where $L_{0}$ is the representative length scale, and periodic boundary conditions were applied in all three directions. The flow cube was discretized uniformly into $N_{g}^{3}$ grid points. The above fluid calculation method is based on the open source of the GFD-Dennou Club library.

Table I lists the computational conditions and flow properties. Four airflow simulations (FLOW-1, 2, 3, and 4) were performed. The airflows have different root-mean-square 
TABLE I. Flow conditions and properties in the DNS.

\begin{tabular}{lccccc}
\hline \hline & Symbol & FLOW-1 & FLOW-2 & FLOW-3 & FLOW-4 \\
\hline Grid number & $N_{g}$ & 64 & 64 & 64 & 96 \\
Velocity rms $\left(\mathrm{m} \mathrm{s}^{-1}\right)$ & $u^{\prime}$ & 0.100 & 0.143 & 0.209 & 0.151 \\
Integral scale $\left(\times 10^{-3} \mathrm{~m}\right)$ & $l_{I}$ & 9.20 & 9.04 & 7.80 & 15.8 \\
Taylor microscale $\left(\times 10^{-3} \mathrm{~m}\right)$ & $l_{\lambda}$ & 5.47 & 4.64 & 3.90 & 6.78 \\
Kolmogorov scale $\left(\times 10^{-4} \mathrm{~m}\right)$ & $l_{\eta}$ & 4.60 & 3.54 & 2.69 & 4.17 \\
Large eddy turn over time $(\mathrm{s})\left(=u^{\prime 2} / \epsilon\right)$ & $T_{e}$ & 0.133 & 0.0951 & 0.0677 & 0.204 \\
Turbulent Reynolds number $\left(=u^{\prime} l_{\lambda} / \nu\right)$ & $\mathrm{Re}_{\lambda}$ & 36.3 & 44.3 & 54.3 & 68.4 \\
Grid resolution & $k_{\max } l_{\eta}$ & 2.94 & 2.27 & 1.72 & 2.00 \\
\hline \hline
\end{tabular}

(rms) values of velocity fluctuation, $u^{\prime}$, which is controlled by the forcing strength $F_{i}$. The number of grid points and the representative length scales for the fluid calculation were set as $N_{g}=64$ and $L_{0}=0.005 \mathrm{~m}$ for FLOW-1, 2, and 3, while $N_{g}=96$ and $L_{0}=0.01 \mathrm{~m}$ were used for FLOW-4. The typical length scales $l_{I}, l_{\lambda}$, and $l_{\eta}$ are the integral scale, the Taylor microscale and the Kolmogorov scale, respectively. They are calculated as

$$
l_{I}=\frac{\pi}{2 u^{\prime 2}} \int_{0}^{\infty} \frac{E(k)}{k} d k, \quad l_{\lambda}=\sqrt{\frac{15 u^{\prime 2} \nu}{\epsilon}}, \quad l_{\eta}=\left(\frac{\nu^{3}}{\epsilon}\right)^{1 / 4},
$$

where $u^{\prime}$ is the rms of the flow velocity fluctuations, $k$ is the wave number, $E(k)$ is the energy spectrum, and $\epsilon$ is the energy dissipation rate. The maximum possible wavenumber, $N_{g} / 2 L_{0}$, is denoted by $k_{\max }$. It is widely accepted that the product $k_{\max } l_{\eta}$ should be greater than unity for sufficient resolution at small scales, and the values in Table I satisfy this criterion.

\section{B. Droplet motion}

Water droplets are considered here as Stokes particles with inertia, governed by the equation

$$
\frac{\partial V_{p, i}}{\partial t}=-\frac{V_{p, i}-U_{i}}{\tau_{p}}+\delta_{i 2} g
$$

where $V_{p, i}$ is the particle velocity, $g$ is the gravitational acceleration, and $\tau_{p}$ is the particle relaxation time defined as $\tau_{p}=(2 / 9)\left(\rho_{p} / \rho_{f}\right)\left(r^{2} / \nu\right)$, where $r$ is the particle radius and $\rho_{p} / \rho_{f}$ is the ratio of the density of the particle material to that of the fluid, set here to $8.43 \times 10^{2}$ at $1 \mathrm{~atm}$ and $298 \mathrm{~K}$. The gravitational acceleration, $g$, in Eq. (3) was set to $9.8 \mathrm{~m} \mathrm{~s}^{-2}$ for the with-gravity case. The second-order AdamsBashforth method was used for time integration. The flow velocity at a droplet position was linearly interpolated from the adjacent grid values. Strictly speaking, the Stokes drag model is not appropriate for water droplets with radii larger than $50 \mu \mathrm{m}$ since the particle Reynolds number then exceeds unity. However, for simplicity all droplets in this study were considered as Stokes particles. If a nonlinear drag model was employed, the gravity effect would be smaller than shown in this study since the nonlinear drag decreases the gravitational settling velocity. The use of nonlinear drag model, therefore, would not change the conclusion of this study as mentioned in Sec. III D. Turbulence modulation by droplets was assumed negligible because of the high droplet dilution.

\section{Collision statistics}

Wang et ll. $^{28}$ formulated the collisions between monodispersed droplets based on the spherical formulation as

$$
\left\langle K_{c}(r, r)\right\rangle=2 \pi R^{2}\left\langle\left|w_{r}\right|\right\rangle g(R),
$$

where $\langle\cdots\rangle$ denotes an ensemble average, $R(=2 r)$ the collision radius, $\left|w_{r}\right|$ the radial relative velocity at contact, and $g(R)$ the radial distribution function (RDF) at contact. The term $g(R)$ represents the preferential concentration effect, which is equal to unity when particles are uniformly distributed. Collision kernels can be obtained from $\left\langle\left|w_{r}\right|\right\rangle$ and $g(R)$ using Eq. (4). However, in order to validate our analysis procedure, we ran separate simulations to calculate (a) the collision kernels $K_{c}$, and (b) the radial relative velocities and RDFs, $\left|w_{r}\right|$ and $g(R)$.

In the simulations to obtain collision kernels, one of the colliding pair of droplets was removed after collision to avoid excess further collisions. ${ }^{35}$ After the background airflow had reached the statistically stationary stage, 4096 monodispersed water droplets were introduced into the flow. After a period exceeding ten times the particle relaxation time, collision detection was then started. Particle volume fractions were so dilute-order $10^{-4}$ at most - that only binary collisions were considered. The particle-particle aerodynamic interactions were neglected and only the geometric collisions considered. Collision kernels were calculated from the temporal change in the total number of particles. Thus, the collision kernel at the $n$th time step, $K_{c}^{n}$, was calculated as

$$
K_{c}^{n}=2 \frac{N^{n-1}-N^{n}}{V_{d} \Delta t}\left(\frac{2 V_{d}}{N^{n-1}+N^{n}}\right)^{2},
$$

where $N$ is the number of particles, $V_{d}\left(=\left(2 \pi L_{0}\right)^{3}\right)$ is the volume of the computational domain, and $\Delta t$ is the time interval. The first fraction on the right hand side of Eq. (5) is the collision frequency during the $n$th time step, $N_{c}^{n}$. The time interval was determined so that each particle would move less than one-fifth of its diameter during that time, and each run was continued until $5 \%$ of the particles were distinguished. Therefore, the time interval and simulation duration were different in different flow conditions and particle sizes. 
The mean collision kernel, $\left\langle K_{c}\right\rangle$, was calculated by time averaging the collision kernels at each time step.

In the simulations for calculating the radial relative velocities and RDFs, particles were allowed to pass through each other (the ghost-particle condition). The mean radial relative velocity at contact, $\left\langle\left|w_{r}\right|\right\rangle$, and the mean RDF at contact, $g(R)$, in Eq. (4) were calculated as follows. If the distance $l$ between a pair of particles was in the range $R-\delta / 2$ $<l<R+\delta / 2$, the pair was regarded as a colliding pair. Here, the value of $\delta$ was fixed at $\delta=0.02 R$, which is the same value as used in Ref. 28. The mean radial relative velocity, $\left\langle\left|w_{r}\right|\right\rangle$, was obtained by time averaging the mean approach velocity of colliding pairs at each time step, $\left\langle\left|w_{r}\right|\right\rangle^{n}$. The RDF at contact at $n$th time step, $g(R)^{n}$, was calculated as

$$
g(R)^{n}=\frac{N_{\text {pair }}^{n} V_{d}}{V_{s} N^{n}\left(N^{n}-1\right) / 2},
$$

where $V_{s}\left(=4 \pi\left[(R+\delta / 2)^{3}-(R-\delta / 2)^{3}\right] / 3\right)$ is the contact volume and $N_{\text {pair }}$ is the number of colliding pairs. The mean RDF at contact, $g(R)$, was calculated by time averaging $g(R)^{n}$. The time interval $\delta t$ was determined so that each particle would move less than one-fifth of $\Delta$ during that time, a much stricter condition than used for the collision kernel calculations. Each run was continued for the same duration as the corresponding collision kernel simulation. Mean collision kernels obtained directly from Eq. (5) were within 3\% of those obtained via Eq. (4) from $\left\langle\left|w_{r}\right|\right\rangle$ and $g(R)$. This validates our procedures for obtaining the collision statistics.

\section{RESULTS AND DISCUSSION}

\section{A. Influence of gravity on turbulent droplet collisions}

Figure 1 shows (a) collision kernels, (b) radial relative velocities, and (c) RDFs for FLOW-3. The collision kernels are normalized by the local shear rate, $\lambda(=\sqrt{\epsilon / \nu})$, and collision radius, and the radial relative velocities by the rms of flow velocity fluctuations, $u^{\prime}\left(=u_{1}^{\prime}=u_{2}^{\prime}=u_{3}^{\prime}\right)$. The error bars show the standard deviation of the results over the five runs. The dotted lines indicate the points where the Stokes number, St $\left[=\tau_{p} / \tau_{\eta}\right.$, where $\tau_{\eta}(=1 / \lambda)$ is the Kolmogorov dissipation time scale], equals to unity. The collision kernels are significantly decreased in the presence of gravity in the range $r / l_{\eta}>0.1$, where $l_{\eta}$ is the Kolmogorov scale, but not where $r / l_{\eta}<0.1$. This tendency is also clear for the radial relative velocities. In contrast, the RDF increases under gravity except for $\mathrm{St}=1$, where it decreases slightly. The change in RDF, however, is almost within the error bars. Woittiez et $a l .{ }^{34}$ reported that RDF is increased in the presence of gravity, which was attributed to the interaction of droplets with the larger scales of turbulence. The interaction becomes significant when $\tau_{p} / T_{I} \sim 0.5$, where $T_{I}$ is the integral time scale defined as $T_{I}=l_{I} / u^{\prime}$. In FLOW-3, $\tau_{p} / T_{I}=0.5$ corresponds to $\mathrm{St}=3.7$, i.e., $r / l_{\eta}=0.14$, where a small bump is seen in the with-gravity case. Wang and Maxey ${ }^{12}$ reported a decrease of a few percent in a measure of local accumulation for $\mathrm{St}=1$, while Onishi and Komori ${ }^{36}$ reported slight increase or decrease around $\mathrm{St}=1$, depending on Reynolds number. The two studies are consistent in view of the insignificant
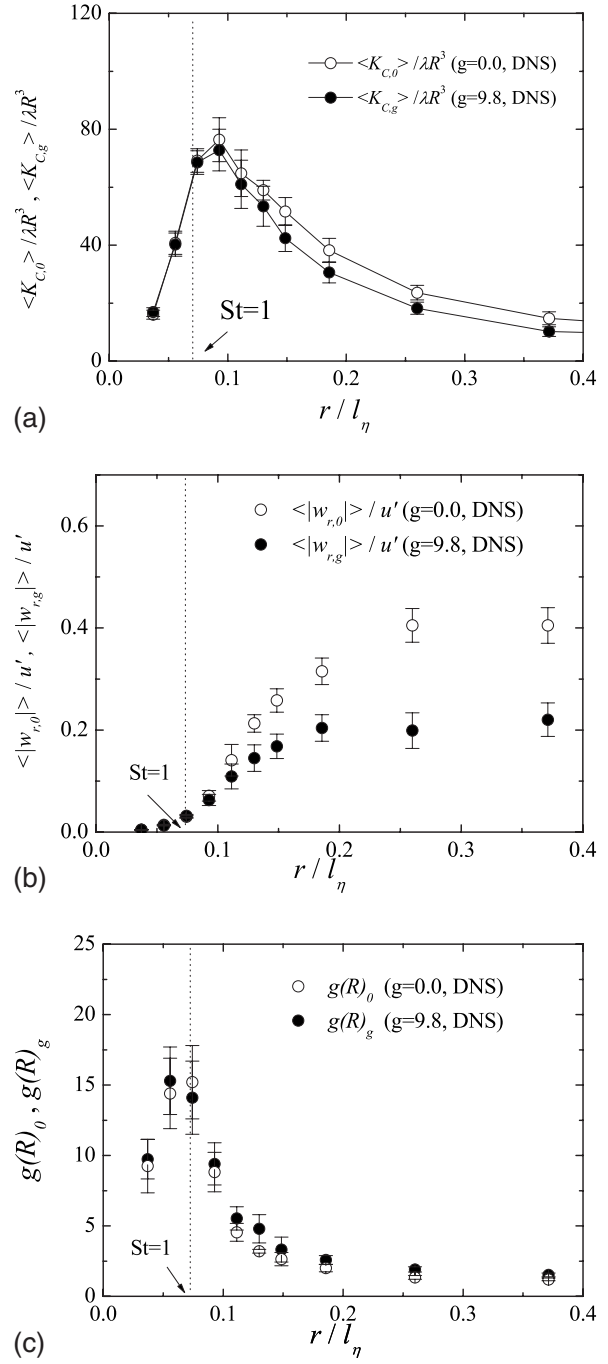

FIG. 1. (a) Collision kernels, (b) radial relative velocities, and (c) RDFs for FLOW-3. Open circles denote the results for the no-gravity case and solid circles for the with-gravity case.

changes in preferential concentrations, though neither discuss the interaction around $\tau_{p} / T_{I} \sim 0.5$. Even if the interaction is significant, however, it will not play an important role in real clouds since $T_{I}$ is as large as $100 \mathrm{~s}$ and $\tau_{p}<0.1 \mathrm{~s}$, giving $\tau_{p} / T_{I}<10^{-3}$. The interaction around $\tau_{p} / T_{I} \sim 0.5$ may be significant in some engineering flows, but not in atmospheric turbulence. This study, therefore, neglects the change in preferential concentration and attributes the decrease in collision kernels to the decrease in radial relative velocities.

\section{B. Modeling of gravitational influence}

The discussion in the previous section implies that

$$
\left\langle K_{c, g}\right\rangle /\left\langle K_{c, 0}\right\rangle \sim\left\langle\left|w_{r, g}\right|\right\rangle /\left\langle\left|w_{r, 0}\right|\right\rangle,
$$

where the subscripts " $g$ " and " 0 " denote the values for the with-gravity case and no-gravity cases, respectively. Assuming a Gaussian distribution of velocity fluctuations, the radial relative velocity at contact, $\left\langle\left|w_{r}\right|\right\rangle$, can be divided into two contributions as 


$$
\left\langle\left|w_{r}\right|\right\rangle=\left[\frac{2}{\pi}\left(\left\langle w_{r, \text { shear }}^{2}\right\rangle+\left\langle w_{r, \text { accel }}^{2}\right\rangle\right)\right]^{1 / 2},
$$

where $w_{r, \text { shear }}$ is the relative velocity due to local fluid shear, and $w_{r, \text { accel }}$ the relative velocity due to turbulent acceleration. The local shear contribution term, $\left\langle w_{r, \text { shear }}^{2}\right\rangle$, can be written as a function of the local shear rate and the collision radius, ${ }^{24}$

$$
\left\langle w_{r, \text { shear }}^{2}\right\rangle=\frac{1}{15} \lambda^{2} R^{2} .
$$

Here, the local shear rate is a fluid turbulence statistic, which is not influenced by gravity. Therefore, $\left\langle w_{r \text {,shear }}^{2}\right\rangle$ is insensitive to gravity. The turbulent acceleration contribution term, $\left\langle w_{r, \text { accel }}^{2}\right\rangle$, can be expanded as

$$
\left\langle w_{r, \text { accel }}^{2}\right\rangle=\left\langle\left(v_{r}^{(2)}-v_{r}^{(1)}\right)^{\prime 2}\right\rangle=2 v_{p}^{\prime 2}\left(1-\rho_{12}\right),
$$

where $v_{r}^{(2)}-v_{r}^{(1)}$ is the relative velocity in one direction between two colliding particles with separation $R, v_{p}^{\prime 2}$ is the particle velocity fluctuation intensity, and $\rho_{12}\left(=\left\langle v_{r}^{(1)} v_{r}^{(2)}\right\rangle / v_{p}^{\prime 2}\right)$ is the correlation coefficient. If $\mathrm{St}$ is small, the coefficient is consistent with the correlation between two fluid elements with separation $R$,

$$
1-\rho_{12}=\left(\frac{R}{l_{\lambda_{f}}}\right)^{2},
$$

where $l_{\lambda_{f}}$ is the longitudinal Taylor microscale, which is related to the transverse Taylor microscale, $l_{\lambda}$, by $l_{\lambda_{f}}=\sqrt{2} l_{\lambda}$. Equation (11) contains no variable that is sensitive to gravity. Even if St is not close to zero, the correlation coefficient $\rho_{12}$ would be strongly influenced by the distance of two colliding particles, i.e., the collision radius. Therefore, we assume that $\rho_{12}$ is insensitive to gravity. We then obtain the simple relation

$$
\left\langle w_{r, \text { accel }, g}^{2}\right\rangle /\left\langle w_{r, \text { accel }, 0}^{2}\right\rangle \sim v_{p, g}^{\prime 2} / v_{p, 0}^{\prime 2} .
$$

The following equation is suggested for the particle velocity fluctuation $v_{p, 0}^{\prime}$ in the no-gravity case, ${ }^{28,33,37}$

$$
\begin{aligned}
v_{p, 0}^{\prime 2}=3 v_{i, 0}^{\prime 2} & =3 u_{i}^{\prime 2} \frac{\gamma}{\gamma-1}\left[\frac{1}{1+\theta_{0}}-\frac{1}{\gamma\left(1+\gamma \theta_{0}\right)}\right] \\
& \equiv 3 u_{i}^{\prime 2} f\left(\theta_{0}\right),
\end{aligned}
$$

where $v_{i, 0}^{\prime}$ is the particle velocity fluctuation in the $x_{i}$-direction, $\theta_{0}=2.5 \tau_{p} / T_{L P, 0}$, where $T_{L P, 0}$ is the Lagrangian particle integral time scale, and the parameter $\gamma$ signifies the Reynolds dependence. Here, $T_{L P, 0}$ is assumed to be the largeeddy turnover time $T_{e}\left(=u^{\prime 2} / \epsilon\right)$ and the parameter $\gamma$ $=0.183 \mathrm{Re}_{\lambda} / \overline{15}^{28}$ The particle velocity fluctuation under gravity, $v_{p, g}^{\prime 2}\left(=3 v_{i, g}^{\prime 2}\right)$, can be written in the same manner,

$$
v_{p, g}^{\prime 2}=3 u_{i}^{\prime 2} f\left(\theta_{g}\right),
$$

where $\theta_{g}=2.5 \tau_{p} / T_{L P, g}$. The Lagrangian particle integral timescale under gravity, $T_{L P, g}$, is smaller than that under the no-gravity case since gravitational sedimentation decreases the interaction time between droplet and fluid. On the basis of this idea, we propose a model for $T_{L P, g}$. The production of the Lagrangian particle integral scale and the mean relative velocity between particle and fluid has a length scale, which corresponds to the flow integral scale. The flow scale is in- sensitive to gravity. The above production is therefore not influenced by gravity; that is, $T_{L P, g}\left\langle\left|V_{p}-U\right|_{g}\right\rangle=T_{L P, 0}\langle| V_{p}$ $\left.-\left.U\right|_{0}\right\rangle$. This leads to

$$
T_{L P, g}=\frac{\left\langle\left|V_{p}-U\right|_{0}\right\rangle}{\left\langle\left|V_{p}-U\right|_{g}\right\rangle} T_{L P, 0} .
$$

Assuming Gaussian distributions of particle and fluid velocity fluctuations, and zero mean velocities of particle and fluid, we obtain the mean particle-fluid relative velocity in the $x_{i}$-direction as

$$
\begin{aligned}
\left\langle\left|V_{p, i}-U_{i}\right|_{0}\right\rangle & =\left[\frac{2}{\pi}\left\langle\left(u_{i}^{\prime}-v_{i, 0}^{\prime}\right)^{2}\right\rangle\right]^{1 / 2} \\
& =\left[\frac{2}{\pi}\left(\left\langle u_{i}^{\prime 2}\right\rangle-\left\langle v_{i, 0}^{\prime 2}\right\rangle\right)\right]^{1 / 2},
\end{aligned}
$$

where we used the fact that particle-fluid correlation, $\left\langle v_{i, 0}^{\prime} u^{\prime}\right\rangle$, is equal to particle self-correlation, $\left\langle v_{i, 0}^{\prime 2}\right\rangle$. In the presence of gravity, the particle velocity in the vertical $\left(x_{2}\right)$ direction is written as the sum of the fluctuation and sedimentation velocities: $V_{p, 2}=v_{2,0}^{\prime}+V_{p, \infty}$. In the same manner, we then obtain $\left\langle\left|V_{p, 2}-U_{2}\right|_{g}\right\rangle=\left[(2 / \pi)\left(\left\langle u_{2}^{\prime 2}\right\rangle-\left\langle v_{2,0}^{\prime 2}\right\rangle+V_{p, \infty}^{2}\right)\right]^{1 / 2}$. Expanding the above estimates of particle-fluid relative velocity into three dimensions, we obtain

$$
T_{L P, g}=\frac{\left\langle\left|V_{p}-U\right|_{0}\right\rangle}{\left\langle\left|V_{p}-U\right|_{g}\right\rangle} T_{L P, 0}=\sqrt{\frac{3\left(1-f\left(\theta_{0}\right)\right)}{3\left(1-f\left(\theta_{0}\right)\right)+\sigma^{2}}} T_{L P, 0},
$$

where $\sigma=V_{p, \infty} / u^{\prime}$ is a nondimensional parameter quantifying the influence of sedimentation. Accordingly, $\theta_{g}$ is given by

$$
\theta_{g}=\sqrt{\frac{3\left(1-f\left(\theta_{0}\right)\right)+\sigma^{2}}{3\left(1-f\left(\theta_{0}\right)\right)}} \theta_{0} .
$$

In summary, we can estimate the gravitational influence on collision kernels (output), $\left\langle K_{c, 0}\right\rangle /\left\langle K_{c, g}\right\rangle$, from the flow and particle parameters. This is the key model proposed in this study, which we envisage being used within the following algorithm:

(1) The collision kernel, $\left\langle K_{c, 0}\right\rangle$, or droplet radial relative velocity in the no-gravity case, $\left\langle\left|w_{r, 0}\right|\right\rangle$, is obtained by DNS or some analytical models (e.g., Ref. 28) from given flow and particle parameters.

(2) $\left\langle w_{r, \text { accel }, 0}^{2}\right\rangle$ is calculated using Eqs. (8) and (9).

(3) The models for particle velocity fluctuations provide an estimate for $v_{p, g}^{\prime 2} / v_{p, 0}^{\prime 2}$, which leads to $\left\langle w_{r, \text { accel }, g}^{2}\right\rangle$ through Eq. (12).

(4) Substitution of $\left\langle w_{r, \text { accel, }, g}^{2}\right\rangle$ into Eq. (8) yields $\left\langle\left|w_{r, g}\right|\right\rangle$.

(5) The estimated $\left\langle\left|w_{r, g}\right|\right\rangle$ and given $\left\langle\left|w_{r, 0}\right|\right\rangle$ then lead to $\left\langle K_{c, g}\right\rangle /\left\langle K_{c, 0}\right\rangle$ through Eq. (7).

\section{Gravitational influence on collision kernels}

Figure 2 shows (a) averaged particle velocity fluctuation intensities and (b) radial relative velocities for FLOW-3. In Fig. 2(a), the solid line shows the model results for particle velocity intensities for the no-gravity case and the broken 

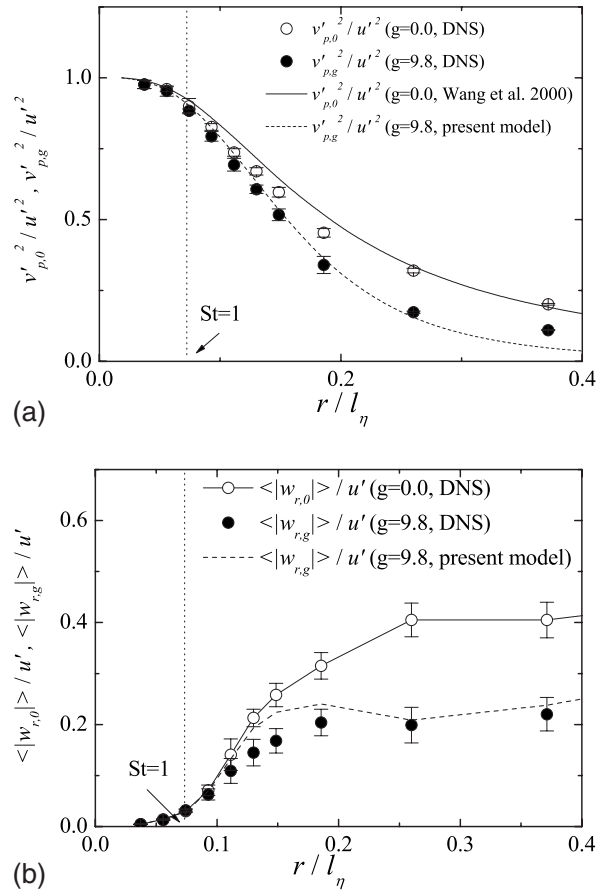

FIG. 2. (a) Averaged particle velocity fluctuation intensities and (b) radial relative velocities for FLOW-3. Symbols are as in Fig. 1. The solid line in (a) is drawn from Eq. (13) and broken line from Eq. (14). The broken line in (b) shows the estimated radial relative velocities under gravity. lines for the with-gravity case. The model results for the no-gravity case are in good agreement with the DNS results for whole range of $r / l_{\eta}$. In the with-gravity case, our model results agree with the DNS results for the limited range of $r / l_{\eta}<0.30$. Beyond this threshold, strong anisotropy of the particle motion invalidates the model of Eq. (18). Similarly, the thresholds for FLOW-1-4 were $r / l_{\eta}=0.13,0.20,0.30$, and 0.17 respectively, which correspond to $\sigma=4.4,4.3,3.8$, and 4.1. These values suggest that our model for the particle velocity fluctuation under gravity is valid for the range $\sigma$ $<4$.

Figure 2(b) shows the DNS results and model estimates for the radial relative velocities for FLOW-3. The overall features of the model estimates for $\left\langle\left|w_{r, g}\right|\right\rangle$ are consistent with the DNS results, though some discrepancies are found around $r / l_{\eta}=0.15$. These discrepancies might be brought about by the simplifications adopted in our model. There are two large simplifications in our model for the gravitational influence: (1) the neglection of the change in preferential concentrations due to the interaction of droplets with the larger scales of turbulence around $\tau_{p} / T_{I} \sim 0.5$ (Ref. 34) and (2) the assumption that the correlation coefficient $\rho_{12}$ in Eq. (10) is insensitive to gravity. Judging from the fact that discrepancies in $\left\langle\left|w_{r, g}\right|\right\rangle$ are found around $\tau_{p} / T_{I} \sim 0.5$-corresponding to $r / l_{\eta}=0.14$ for FLOW-3-the discrepancies are possibly related to the former simplification. The interaction of droplets with the larger scales of turbulence may influence the droplet relative motions as well

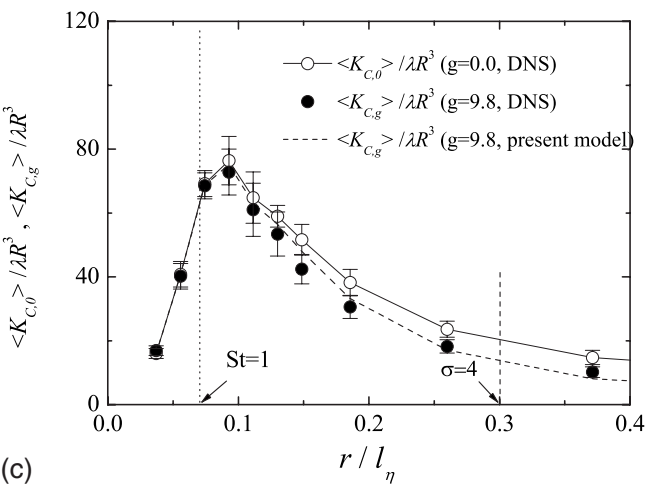

(a)

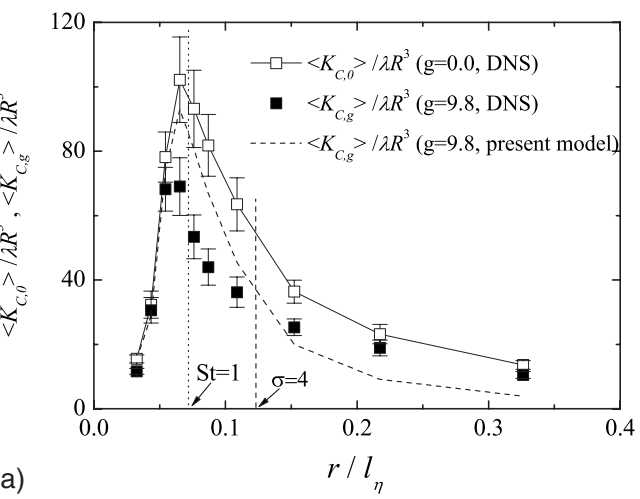

(c)

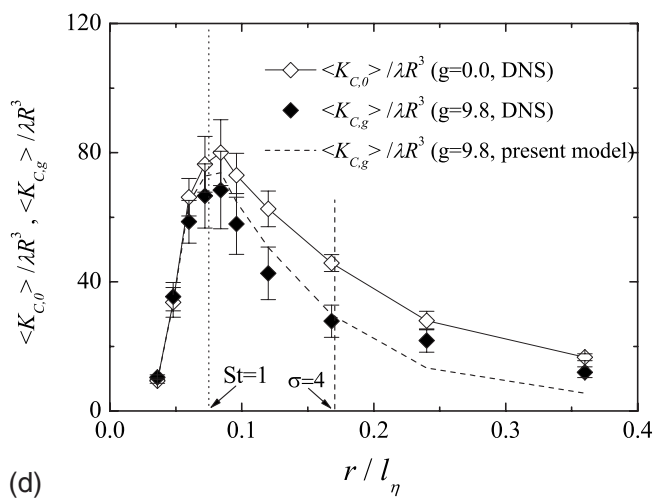

FIG. 3. Comparison of the normalized collision kernel between the model predictions and the DNS results in (a) FLOW-1, (b) FLOW-2, (c) FLOW-3, and (d) FLOW-4. Open symbols denote the collision kernels for the no-gravity case and solid symbols in the with-gravity case. The broken lines are the model results of collision kernels under gravity. 


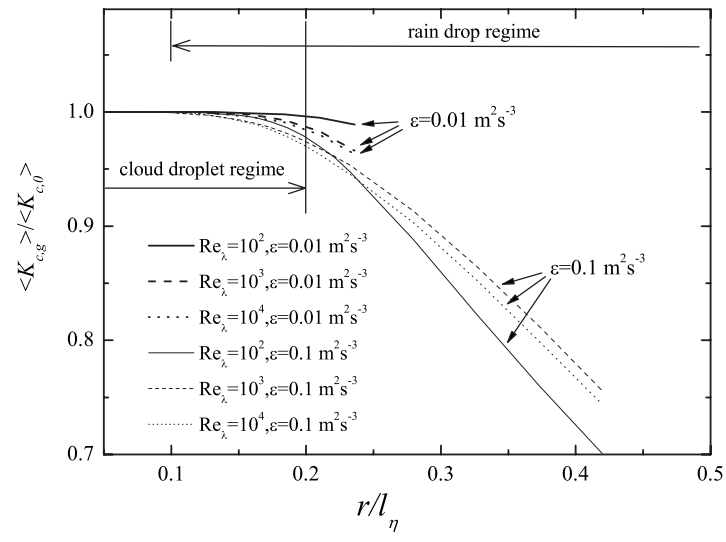

FIG. 4. Model estimations of gravitational influence on collision kernels in flows with the fixed flow velocity fluctuation of $1 \mathrm{~m} \mathrm{~s}^{-1}$, with different energy dissipation rate, $\epsilon$, and turbulent Reynolds numbers, $\operatorname{Re}_{\lambda}$. Thick lines show the results for $\epsilon=0.01 \mathrm{~m}^{2} \mathrm{~s}^{-3}$ and thin lines for $\epsilon=0.1 \mathrm{~m}^{2} \mathrm{~s}^{-3}$.

as the preferential concentrations. If this is true, we can ignore the discrepancies at $\tau_{p} / T_{I} \sim 0.5$ since $\tau_{p} / T_{I}$ is less than $10^{-3}$ for cloud droplets in cloud turbulence.

Figure 3 shows the differences in collision kernels due to gravity in FLOW-1, 2, 3, and 4. The broken lines show the model results for collision kernels in the with-gravity case, $\left\langle K_{c, g}\right\rangle$, obtained by multiplying the model results for $\left\langle K_{c, g}\right\rangle /\left\langle K_{c, 0}\right\rangle$ and the DNS results for $\left\langle K_{c, 0}\right\rangle$. The collision kernel decreases in the presence of gravity, except for a small reversal near $\mathrm{St}=1$ in FLOW-2, where the increase in RDF slightly overcomes the decrease in droplet radial relative velocity. Only Fig. 3(a) shows a significant discrepancy between the model and DNS results for a wide range of $r / l_{\eta}$ values. This discrepancy is caused by the characteristic features of low Reynolds number flows. Wang et al. ${ }^{28}$ found that the small scale separation between energy-containing-scale motions and dissipation-scale motions causes interference between them in low Reynolds number flows causing the discrepancy between the characteristics of particle collisions in low and high Reynolds number flows. In such situations, the assumption behind Eq. (8) may become invalid. For the other three flows (FLOW-2, 3, and 4), the model results agree with the DNS results for the range $\sigma<4$, where our model for $v_{p, g}^{\prime}$ is valid. These results confirm the validity of our model for $\left\langle K_{c, g}\right\rangle /\left\langle K_{c, 0}\right\rangle$.

\section{Gravitational influence on collision kernels in high Reynolds number flows}

In convective clouds, flow velocity fluctuation values, $u^{\prime}$, are typically around $1 \mathrm{~m} \mathrm{~s}^{-1}$, with energy dissipation rate, $\epsilon$, of order $10^{-2}$ (shallow cumulus) to $10^{-1} \mathrm{~m}^{2} \mathrm{~s}^{-3}$ (deep cumulus) and $\operatorname{Re}_{\lambda}$ of the order from $10^{3}$ to $10^{4}$ (e.g., Refs. 10 and 28). Here we fix $u^{\prime}=1 \mathrm{~m} \mathrm{~s}^{-1}$ and discuss the gravitational influence on collisions of monodispersed water droplets in different flow conditions.

Figure 4 shows the model results for $\left\langle K_{c, g}\right\rangle /\left\langle K_{c, 0}\right\rangle$. The model of Wang et al. ${ }^{28}$ was used for calculating the radial relative velocities in the no-gravity case, $\left\langle\left|w_{r, 0}\right|\right\rangle$, as an input to our model. The horizontal axis shows the normalized droplet size, $r / l_{\eta}$. Cloud droplet radii are typically tens of microns, and the Kolmogorov scale in the atmosphere ranges from $500 \mu \mathrm{m}$ to $1 \mathrm{~mm}$. Thus, the cloud droplet regime corresponds to the range $r / l_{\eta}<0.2$. As discussed in Fig. 2(a), strong anisotropy of droplets invalidates the model for $v_{p, g}^{\prime}$ in the range of $\sigma>4$. The threshold of $\sigma=4$ corresponds to $r / l_{\eta}=0.24$ for $\epsilon=0.01 \mathrm{~m}^{2} \mathrm{~s}^{-3}$, and $r / l_{\eta}=0.42$ for $\epsilon$ $=0.1 \mathrm{~m}^{2} \mathrm{~s}^{-3}$; the cloud droplet regime of $r / l_{\eta}<0.2$ exceeds neither thresholds. In the cloud droplet regime we are targeting in this study, the decrease in collision kernel is larger for larger $\epsilon$, and for larger $\operatorname{Re}_{\lambda}$. However, the maximum decrease is at most $3 \%\left(\right.$ at $\left.r / l_{\eta}=0.2\right)$ even in the flow with $\epsilon$ $=0.1 \mathrm{~m}^{2} \mathrm{~s}^{-3}$ and $\operatorname{Re}_{\lambda}=10^{4}$. Therefore, we can conclude that the gravitational influence on collisions of cloud droplets is negligible in convective clouds. This study employs the Stokes drag model for the droplet motion for simplicity. If a nonlinear drag model was employed, the gravity effect for large droplets would be smaller since the nonlinear drag decreases the gravitational settling velocity. The negligibly small effect of gravity stated in the above conclusion, therefore, is not changed by the nonlinearity of drag.

In the rain drop regime $r / l_{\eta}>0.1$, the decrease due to gravity is larger. For rain drops, collisions with cloud droplets (i.e., collisions between bidispersed particles) are more important than those between monodispersed particles. The gravitational influence on collisions of bidispersed particles has to be investigated further for the rain drop regime.

\section{CONCLUSIONS}

There are two kinds of gravity contributions to collisions. In the presence of gravity, collisions between different-sized droplets are caused by the difference in their settling velocities (differential settling contribution). Gravitational sedimentation influences the droplet-fluid interaction, and consequently changes the collision frequency (gravitational aerodynamic contribution). Collisions between monodispersed droplets are induced by a combination of turbulent collisions and the gravitational aerodynamic contribution, while those between bidispersed droplets additionally include the differential settling contribution.

This paper studies the gravitational aerodynamic contribution to collisions of monodispersed droplets in homogeneous isotropic turbulence by means of direct numerical simulations (DNSs). The DNS has been carried out for four flow conditions and for different particle sizes. The DNS results show that, in certain Stokes and Reynolds regimes, collision frequencies are significantly reduced in the presence of gravity. The droplet relative velocity also decreases, while the RDF increases slightly. Therefore, the decrease in collision frequency is attributed to the decrease in droplet relative velocity. A possible explanation of the decrease in relative velocity is that droplet sedimentation due to gravity shortens the droplet-fluid interaction time, consequently weakening the relative motions between droplets. On the basis of this idea, we developed a model that can estimate the velocity fluctuation of a sedimenting particle under gravity, $v_{p, g}^{\prime}$. Comparisons between the model and DNS results con- 
firmed the validity of the model when the ratio of droplet sedimentation to fluid velocity fluctuation, $\sigma\left(=V_{p, \infty} / u^{\prime}\right)$, is less than four. Beyond $\sigma=4$, strong anisotropy of particle motion begins to invalidate the model.

Utilizing the model for $v_{p, g}^{\prime}$, we constructed a further model for estimating the ratio $\left\langle K_{c, g}\right\rangle /\left\langle K_{c, 0}\right\rangle$ of collision kernels for the with-gravity and no-gravity cases from given flow and particle parameters. Past studies note simply that the gravitational influence is negligible when the droplet sedimenting velocity is much smaller than the flow velocity fluctuation, i.e., $\sigma \ll 1$. In contrast, our model can estimate the gravitational influence on collisions of droplets with $\sigma$ $<4$ considering the Reynolds dependency. The model estimates suggest that the gravitational influence on collisions of cloud droplets is negligible even in the high Reynolds number flows typically found in convective clouds.

One important implication from the present results is that we can neglect the gravitational aerodynamic contribution for collisions between cloud droplets. This supports the existing collision kernel models for monodispersed cloud droplets (e.g., Refs. 28 and 32), which neglects the gravitational aerodynamic contribution.

\section{ACKNOWLEDGMENTS}

This research was supported by Core Research for Evolutional Science and Technology (CREST) Program "Advanced Model Development and Simulations for Disaster Countermeasures" of Japan Science and Technology Agency (JST). The simulations were carried out by using a parallel supercomputer of the Center for Global Environment Research, National Institute for Environmental Studies, Environmental Ministry of Japan.

${ }^{1}$ L. P. Wang, Y. Xue, O. Ayala, and W. W. Grabowski, "Effects of stochastic coalescence and air turbulence on the size distribution of cloud droplets," Atmos. Res. 82, 416 (2006).

${ }^{2}$ A. M. Blyth, "Entrainment in cumulus clouds," J. Appl. Meteorol. 32, 626 (1993).

${ }^{3}$ S. K. Krueger, C.-W. Su, and P. A. Mcmurtry, "Modeling entrainment and finescale mixing in cumulus clouds," J. Atmos. Sci. 54, 2697 (1997).

${ }^{4}$ Y. Yin, Z. Levin, T. G. Reisin, and S. Tzivion, "The effects of giant cloud condensation nuclei on the development of precipitation in convective clouds: A numerical study," Atmos. Res. 53, 91 (2000).

${ }^{5}$ S. C. van den Heever and W. R. Cotton, "Urban aerosol impacts on downwind convective storms," J. Appl. Meteorol. 46, 828 (2007).

${ }^{6}$ R. A. Shaw, W. C. Reade, L. R. Collins, and J. Verlinde, "Preferential concentration of cloud droplets by turbulence: Effects on the early evolution of cumulus cloud droplet spectra," J. Atmos. Sci. 55, 1965 (1998).

${ }^{7}$ P. R. Jonas, "Turbulence and cloud microphysics," Atmos. Res. 40, 283 (1996).

${ }^{8}$ M. B. Pinsky and A. P. Khain, "Turbulence effects on droplet growth and size distributions in cloud: A review," J. Aerosol Sci. 28, 1177 (1997).

${ }^{9}$ P. A. Vaillancourt and M. K. Yau, "Review of particle-turbulence interactions and consequences for cloud physics," Bull. Am. Meteorol. Soc. 81, 285 (2000)

${ }^{10}$ R. A. Shaw, "Particle-turbulence interactions in atmospheric clouds," Annu. Rev. Fluid Mech. 35, 183 (2003).

${ }^{11}$ A. P. Khain, M. B. Pinsky, T. Elperin, N. Kleeorin, I. Rogachevski, and A. Kostinski, "Critical comments to results of investigations of drop collisions in turbulent clouds," Atmos. Res. 86, 1 (2007).
${ }^{12}$ L. P. Wang and M. R. Maxey, "Settling velocity and concentration distribution of heavy particles in homogeneous isotropic turbulence," J. Fluid Mech. 256, 27 (1993)

${ }^{13}$ C. Y. Yang and U. Lei, "The role of the turbulent scales in the settling velocity of heavy particles in homogeneous isotropic turbulence," J. Fluid Mech. 371, 179 (1998).

${ }^{14}$ J. Dávila and J. C. R. Hunt, "Settling of small particles near vortices and in turbulence," J. Fluid Mech. 440, 117 (2001).

${ }^{15}$ K. D. Squires and J. K. Eaton, "Measurements of particle dispersion obtained from direct numerical simulations of isotropic turbulence," J. Fluid Mech. 226, 1 (1991).

${ }^{16}$ S. Sundaram and L. R. Collins, "Collision statistics in an isotropic particle-laden turbulent suspension. Part 1. Direct numerical simulations," J. Fluid Mech. 335, 75 (1997).

${ }^{17}$ W. C. Reade and L. R. Collins, "Effect of preferential concentration on turbulent collision rates," Phys. Fluids 12, 2530 (2000).

${ }^{18}$ L. P. Wang, O. Ayala, S. E. Kasprzak, and W. W. Grabowski, "Theoretical formulation of collision rate and collision efficiency of hydrodynamically interacting cloud droplets in turbulent atmosphere," J. Atmos. Sci. 62, 2433 (2005).

${ }^{19}$ L. P. Wang, O. Ayala, and W. W. Grabowski, "Effect of aerodynamic interactions on the motion of heavy particles in a bidisperse suspension," J. Turbul. 8, N25 (2007).

${ }^{20}$ M. B. Pinsky and A. P. Khain, "Collision of cloud droplets in a turbulent flow. Part IV. Droplet hydrodynamic interactions," J. Atmos. Sci. 64, 2462 (2007).

${ }^{21}$ C. N. Franklin, P. A. Vaillancourt, M. K. Yau, and P. Bartello, "Collision rates of cloud droplets in turbulent flow," J. Atmos. Sci. 62, 2451 (2005).

${ }^{22}$ C. N. Franklin, P. A. Vaillancourt, and M. K. Yau, "Statistics and parameterizations of the effect of turbulence on the geometric collision kernel of cloud droplets," J. Atmos. Sci. 64, 938 (2007).

${ }^{23}$ S. Ghosh, J. Davila, J. C. R. Hunt, A. Srdic, H. J. S. Fernando, and P. R. Jonas, "How turbulence enhances coalescence of settling particles with application to rain in clouds," Proc. R. Soc. London, Ser. A. 461, 3059 (2005).

${ }^{24}$ P. G. Saffman and J. S. Turner, "On the collision of drops in turbulent clouds," J. Fluid Mech. 1, 16 (1956).

${ }^{25}$ L. P. Wang, A. S. Wexler, and Y. Zhou, "On the collision rate of small particles in isotropic turbulence. I. Zero-inertia case," Phys. Fluids 10, 266 (1998).

${ }^{26} \mathrm{Z}$. Dodin and T. Elperin, "On the collision rate of particles in turbulent flow with gravity," Phys. Fluids 14, 2921 (2002).

${ }^{27}$ W. W. Grabowski and P. A. Vaillancourt, "Comments on preferential concentration of cloud droplets by turbulence: Effects on the early evolution of cloud droplet spectra," J. Atmos. Sci. 56, 1433 (1999).

${ }^{28}$ L. P. Wang, A. S. Wexler, and Y. Zhou, "Statistical mechanical description and modelling of turbulent collision of inertial particles," J. Fluid Mech. 415, 117 (2000)

${ }^{29}$ Y. Zhou, A. S. Wexler, and L. P. Wang, "Modelling turbulent collision of bidisperse inertial particles," J. Fluid Mech. 433, 77 (2001).

${ }^{30}$ L. P. Wang, O. Ayala, Y. Xue, and W. W. Grabowski, "Comments on 'Droplets to drops by turbulent coagulation'," J. Atmos. Sci. 63, 2397 (2006).

${ }^{31}$ N. Riemer and A. S. Wexler, "Droplets to drops by turbulent coagulation," J. Atmos. Sci. 62, 1962 (2005).

${ }^{32} \mathrm{R}$. Onishi, "Numerical simulations of chemical reaction and droplet growth in environmental turbulent flows," Ph.D. thesis, 2005.

${ }^{33}$ J. J. E. Williams and R. I. Crane, "Particle collision rate in turbulent flow," Int. J. Multiph. Flow 9, 421 (1983).

${ }^{34}$ E. J. P. Woittiez, H. J. J. Jonker, and L. M. Portela, "On the combined effects of turbulence and gravity on droplet collisions in clouds: A numerical study." J. Atmos. Sci. 66, 1926 (2009).

${ }^{35}$ Y. Zhou, A. S. Wexler, and L. P. Wang, "On the collision rate of small particles in isotropic turbulence. II. Finite inertia case,” Phys. Fluids 10, 1206 (1998).

${ }^{36} \mathrm{R}$. Onishi and S. Komori, "The collision rate of monodisperse particles in turbulent flows with gravity," Adv. Fluid. Mech. 5, 229 (2004).

${ }^{37}$ F. E. Kruis and K. A. Kusters, "The collision rate of particles in turbulent flow," Chem. Eng. Commun. 158, 201 (1997). 